\title{
PREDICTIVE FUNCTIONAL CONTROL OF INTEGRATING PROCESS BASED ON IMPULSE RESPONSE
}

\author{
ZHANG Bin ${ }^{1}$, LI Ping ${ }^{2}$, ZHANG Wei-dong ${ }^{1}$ \\ 1.Department of Automation, Shanghai Jiaotong University, Shanghai 200030, China \\ 2. Information Engineering Faculty, Liaoning University of Petroleum \& Chemical \\ Technology, Liaoning Fushun 113001, China
}

\begin{abstract}
The predictive model is built according to the characteristics of the impulse response of integrating process. In order to eliminate the permanent offset between the setpoint and the process output in the presence of the load disturbance, a novel error compensation method is proposed. Then predictive functional control of integrating process is designed. The method given generates a simple control structure, which can significantly reduce online calculation. Moreover, the tuning of the controller is fairly straightforward. Simulation results indicate that the designed control system is relatively robust to the parameters variation of the process. Copyright $\odot 2005$ IFAC
\end{abstract}

Keywords: integrating process, time delay, impulse response, predictive functional control, error compensation.

\section{INTRODUCTION}

There are some process control systems where the dynamics contain integration or very long time constants. Noteworthy in the integrating processes is the poles at the original point which characterize open loop instability, which indicates that a bounded input will lead to unbounded output and, hence, saturation. The combined effects of poles at the original point and the time delay make the design task very difficult (Zhang, et al., 1999).

For the predictive control, the appropriate model selection is helpful for the controller design. It is well known that non-parameter model plays an important role to model process behaviour, such as step response model and impulse response model are adopted in Dynamic Matrix Control (DMC) (Cutler and Ramaker, 1980) and Model Algorithm Control (MAC) (Rouhani and Mehra, 1982), respectively, which have contributed largely to the development of model predictive control. Zhang (2001) presented a predictive control algorithm of integrating process based on step response model. However impulse response can be obtained easily for integrating process, which is shown in Fig. 1, where $s_{i}$ are the coefficients of open loop impulse response at sampling time $i$. Note that $s_{i}$ keep the value of $s_{N}$ at certain time $i>N$, thus it is easy to construct integrating process model with impulse response.

Predictive functional control (PFC) (Richalet, 1993; Ernst et al., 1996) is a new model predictive control, it is one such algorithm which achieves computational simplicity by using simpler but more intuitive design guidelines (Rossiter and Richalet,
2002). PFC has the following advantages: less calculation on line, simpler algorithm and higher control precision. These merits make it achieve wide success in industrial applications. In this paper, PFC approach is applied for integrating process control, the controller design is based on the impulse response model and a novel error compensation method is introduced to eliminate the permanent offset between the setpoint and the process output, which is also contributed to improve the disturbance rejection. Simulations are given to validate the effectiveness and robustness of the proposed algorithm.

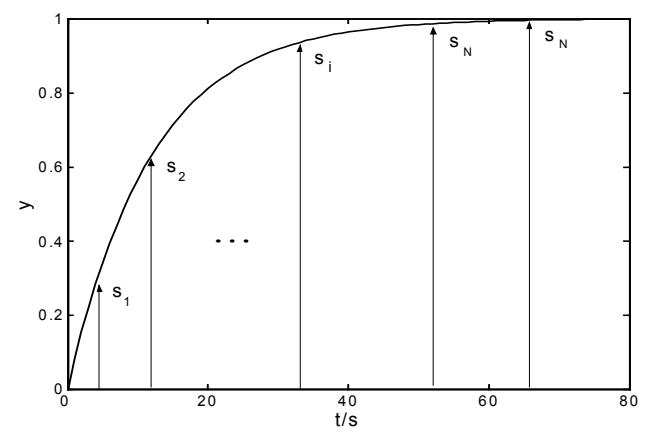

Fig. 1. Open loop impulse response of integrating process.

\section{PREDICTIVE FUNCTIONAL CONTROL OF INTEGRATING PROCESS BASED ON IMPLUSE RESPONSE}

PFC belongs to the classical family of Model Based Predictive Control since it contains the following four basic generic principles: 
.Internal model: used for prediction

.Reference trajectory: to specify the property of the closed loop system

.Structured manipulated variable and controller algorithm

.Model error compensation to take into account prediction error

\subsection{Internal model.}

For PFC algorithm, any type of model can be used. Assume a linear, single pole at the original point, SISO integrating process with finite gain, described by an infinite impulse response model

$$
y_{m}(k)=\sum_{i=1}^{\infty} s_{i} u(k-i-d)
$$

where $d$ satisfies $T_{d}=d T_{s}$, and $T_{d}$ is a time delay of integrating process, $T_{s}$ is the sampling time. Note that $s_{i}$ keep the value of $s_{N}$ at certain time $i>N$. Therefore

$$
\begin{aligned}
& y_{m}(k)=\sum_{i=1}^{\infty} s_{i} u(k-i-d) \\
& =\sum_{i=1}^{N} s_{i} u(k-i-d)+s_{N} \sum_{i=1}^{\infty} u(k-N-i-d)
\end{aligned}
$$

Above equation can be transformed into the $Z$ domain to give the following equation

$$
y_{m}(z)=\sum_{i=1}^{N} s_{i} z^{-i-d} u(z)+s_{N} z^{-1-d-N} \frac{1}{1-z^{-1}} u(z)
$$

By the transformation of (3), the impulse response model of integrating process is presented with the following form

$$
y_{m}(k)=y_{m}(k-1)+\sum_{i=1}^{N}\left(s_{i}-s_{i-1}\right) u(k-i-d)
$$

where $s_{0}=0$.

\subsection{Reference trajectory.}

Reference trajectory specifies the dynamic path over the predictive horizon $\left[0, H_{i}\right]$ in the future time that process output has to rally the setpoint. PFC computes future process input such that the predicted process output follows the reference trajectory. Therefore, the reference trajectory specifies the property of the closed loop system. For many applications it is sufficient to describe it by the firstorder exponential reference trajectory

$$
y_{\text {ref }}(k+i)=w(k+i)-\lambda^{i}\left(w(k)-y_{p}(k)\right)
$$

where $\lambda=\exp \left(-T_{s} / T_{r e f}\right), T_{r e f}$ is the desired response time of the closed loop system, $y_{p}$ is the process output, $w$ is the setpoint.

\subsection{Predicted output and structured manipulated variable.}

PFC considers that prediction $y_{m}(k+i)$ made by the internal model can be divided into two components (Ernst et al., 1996 ):

$$
y_{m}(k+i)=y_{f r}(k+i)+y_{f o}(k+i)
$$

.Free response $y_{f r}(k+i)$ is the model response assuming $u(k+i)=0(i \geq 0)$, therefore it depends on the past manipulated variables and actual model output $y_{m}(k)$.

.Forced response $y_{f o}(k+i)$ is the model output depending on the set of future manipulated variables $u(k+i)(i \geq 0)$.

PFC is different from other model predictive control algorithms. Instead of calculating control signal without restrictions, which may result in a wild control signal, PFC adopts structured future manipulated variables, which considers that the future manipulated variables are parameterized by $n_{B}$ prior known base functions $u_{B j}$.

$$
u(k+i)=\sum_{j=1}^{n_{B}} \mu_{j}(k) u_{B j}(i) i=0,1, \cdots, H-1
$$

Each base function $u_{B j}$ induces a base output $y_{B j}(i)$ known by predictive model in advance. The forced response can be expressed as a linear combination of the function $y_{B j}(i)$

$$
y_{f o}(k+i)=\sum_{j=1}^{n_{B}} \mu_{j}(k) y_{B j}(i) \quad i=1, \cdots, H-1
$$

where $\mu_{j}(k), j \in\left[1, n_{B}\right] \quad$ are the unknown coefficients. The selection of the base functions is driven by the nature of the setpoint, it is usually selected as polynomial, sine and exponential.

Using (4), at sampling time $k+H_{i}$ inside the optimization horizon, the output can be predicted by the following equation

$$
\begin{gathered}
y_{m}\left(k+H_{i}\right)=y_{f r}\left(k+H_{i}\right)+y_{f o}\left(k+H_{i}\right) \\
y_{f o}\left(k+H_{i}\right)=\boldsymbol{U}_{H_{i}} \boldsymbol{S}_{H_{i}}{ }^{T}
\end{gathered}
$$

$y_{f r}\left(k+H_{i}\right)=$

$$
\boldsymbol{U}_{1 H_{i}} \boldsymbol{S}_{1 H_{i}}{ }^{T}+\boldsymbol{U}_{2 H_{i}} \boldsymbol{S}_{2 H_{i}}{ }^{T}+\boldsymbol{U}_{3 H_{i}} \boldsymbol{S}_{3 H_{i}}{ }^{T}+y_{m}(k-1)
$$

where

$$
\begin{aligned}
& \boldsymbol{S}_{H_{i}}=\left[\begin{array}{llll}
s_{1} & s_{2} & \cdots & s_{H_{i}-d}
\end{array}\right] \text {, } \\
& \boldsymbol{U}_{H_{i}}=\left[u\left(k+H_{i}-1-d\right) u\left(k+H_{i}-2-d\right) \cdots u(k)\right] \text {, } \\
& \boldsymbol{S}_{1 H_{i}}=\left[\begin{array}{llll}
s_{H_{i}-d+1} & s_{H_{i}-d+2} & \ldots & s_{H_{i}+1}
\end{array}\right] \text {, } \\
& \boldsymbol{U}_{1 H_{i}}=\left[\begin{array}{llll}
u(k-1) & u(k-2) & \cdots & u(k-d-1)
\end{array}\right], \\
& \boldsymbol{S}_{2 H_{i}}=\left[\begin{array}{ll}
s_{H_{i}+2}-s_{1} & s_{H_{i}+3}-s_{2} \cdots s_{N}-s_{N-H_{i}-1}
\end{array}\right] \text {, } \\
& \boldsymbol{U}_{2 H_{i}}=\left[\begin{array}{lll}
u(k-d-2) & u(k-d-3) \cdots u\left(k-d-N+H_{i}\right)
\end{array}\right] \\
& \boldsymbol{S}_{3 H_{i}}=\left[\begin{array}{ll}
s_{N}-s_{N-H_{i}} & s_{N}-s_{N-H_{i}+1} \ldots s_{N}-s_{N-1}
\end{array}\right] \text {, } \\
& \boldsymbol{U}_{3 H_{i}}= \\
& {\left[u\left(k-N-d-1+H_{i}\right) u\left(k-N-d-2+H_{i}\right) \cdots u(k-N-d)\right] .}
\end{aligned}
$$

\subsection{Model error compensation.}

PFC is a closed loop control algorithm. In practical, model predictive output always differs from the actual process output due to model mismatch and disturbance influence. There are several methods to 
eliminate the permanent offset by compensating the reference trajectory with predictive error between model output and process output at each time instant of the coincidence horizon. For PFC algorithm of the stable process, a flat prediction of the error $e(k+i)$ is usually adopted, that is $e(k+i)=e(k)=y_{p}(k)-y_{m}(k)$. However for integrating process the method of flat prediction of the error compensation may cause a non-zero steadystate error in the presence of the load disturbance. Here a novel error compensation method is proposed, which can eliminate the steady-state error caused by load disturbance, it follows:

$$
e(k+i)=e(k)+i[e(k)-e(k-1)]
$$

\subsection{Optimization calculation.}

PFC algorithm adopts an online optimizing method. A quadratic performance index may be adopted, $\boldsymbol{U}_{H_{i}}$ is calculated by minimizing the sum of the quadratic difference between the predicted process output and reference trajectory at all coincidence points. The criterion takes the following form

$$
\text { Min } J_{p}=\sum_{i=H_{1}}^{H_{2}}\left[y_{r e f}(k+i)-y_{m}(k+i)-e(k+i)\right]^{2}
$$

Substituting (9) and (12) into (13), the unknown parameters are $\mu_{j}(k)\left(j=1,2, \cdots, n_{B}\right)$, thus online computation can be reduced significantly. Then the future manipulated variables $u(k+i)$ can be derived according to (7). However only the first term $u(k)$ is effectively applied for the control.

\subsection{The control law equation.}

While the value of changing rate for the setpoint is less than or equal to $\theta$, only one base function, that is $u_{B j}=1$, can be adopted for constructing manipulated variables.

\section{By (7) we obtain}

$$
\mu_{1}(k)=u(k+j)=u(k) \quad j=1, \cdots, H_{i}-1
$$

Substituting (14) into (13), the only unknown parameter is $\mu_{1}(k)$, then $H_{1}=H_{2}=H$ may be chosen to calculate $u(k)$. Let

$$
\frac{\partial J_{p}}{\partial \mu_{1}(k)}=0
$$

The optimal manipulated variable is obtained by solving (15)

$$
\begin{array}{r}
\mu_{1}(k)=u(k)=\left[y_{r e f}\left(k+H_{1}\right)-\boldsymbol{S}_{1 H_{1}} \boldsymbol{U}^{T}{ }_{1 H_{1}}-\boldsymbol{S}_{2 H_{1}} \boldsymbol{U}^{T}{ }_{2 H_{1}}-\right. \\
\frac{\left.\boldsymbol{S}_{3 H_{1}} \boldsymbol{U}^{T}{ }_{3 H_{1}}-\left(H_{1}+1\right) e(k)+H_{1} e(k-1)-y_{m}(k-1)\right]}{\boldsymbol{I} \boldsymbol{S}_{H_{1}}}
\end{array}
$$

where $y_{\text {ref }}\left(k+H_{1}\right)$ is shown in (5), $\boldsymbol{I}$ is an unit row vector.
While the value of changing rate for the setpoint is greater than $\theta$, that is a ramp tracking, two base functions may be selected to construct manipulated variables, which are step response function and ramp function shown as follows

$$
u(k+j)=\mu_{1}(k)+\mu_{2}(k) j \quad j=0,1 \cdots, H_{i}-1
$$

where $\mu_{1}(k)$ and $\mu_{2}(k)$ are unknown coefficients. In order to obtain the coefficients $\mu_{1}(k)$ and $\mu_{2}(k)$, it requires that at least two coincidence points $H_{1}$ and $H_{2}$ should be selected. Therefore (13) can be rewritten as

$$
\begin{gathered}
\operatorname{Min} J_{P}=\left[y_{\text {ref }}\left(k+H_{1}\right)-y_{m}\left(k+H_{1}\right)-e\left(k+H_{1}\right)\right]^{2}+ \\
{\left[y_{\text {ref }}\left(k+H_{2}\right)-y_{m}\left(k+H_{2}\right)-e\left(k+H_{2}\right)\right]^{2}}
\end{gathered}
$$

where $y_{m}\left(k+H_{i}\right)$ and $y_{\text {ref }}\left(k+H_{i}\right)(i=1,2)$ are the model predicted output and the reference trajectory, respectively.

$$
\begin{aligned}
& e\left(k+H_{1}\right)=e(k)+H_{1}[e(k)-e(k-1)] \\
& e\left(k+H_{2}\right)=e(k)+H_{2}[e(k)-e(k-1)]
\end{aligned}
$$

Substituting (17) into (10), we obtain that

$$
\begin{aligned}
& y_{f o}\left(k+H_{i}\right)=\sum_{j=1}^{H_{i}-d} s_{j} u\left(k+H_{i}-j-d\right) \\
& =\mu_{1}(k) \boldsymbol{S}_{H_{i}} \boldsymbol{I}_{i}^{T}+R_{i} \mu_{2}
\end{aligned}
$$

where $R_{i}=\sum_{j=1}^{H_{i}-d} s_{j}\left(H_{i}-j-d\right), \quad i=1,2$.

Substitute (19), (20) and (5) into (18), let

$$
\frac{\partial J_{p}}{\partial \mu_{1}(k)}=0, \frac{\partial J_{p}}{\partial \mu_{2}(k)}=0
$$

Manipulated variable is obtained by solving (21)

$$
\mu_{1}(k)=u(k)=\frac{A_{1} R_{2}-A_{2} R_{1}}{S_{11} R_{2}-S_{22} R_{1}}
$$

where $S_{i i}=\boldsymbol{I}_{i} \boldsymbol{S}_{H_{i}}{ }^{T}, \boldsymbol{I}_{i}(i=1,2)$ is an unit row vector

$$
\begin{aligned}
A_{i}= & y_{r e f}\left(k+H_{i}\right)-\boldsymbol{S}_{1 H_{i}} \boldsymbol{U}_{1 H_{i}}{ }^{T}-\boldsymbol{S}_{2 H_{i}} \boldsymbol{U}_{2 H_{i}}{ }^{T}-\boldsymbol{S}_{3 H_{i}} \boldsymbol{U}_{3 H_{i}}{ }^{T} \\
& -y_{m}(k-1)-H_{i}(e(k)-e(k-1))-e(k) .
\end{aligned}
$$

Explicit control input constraints is not directly handled in this paper, when input and/or state related constraints need to be considered, the technique proposed by $\mathrm{Abu}$ el Ata-Doss can be adopted.

\section{DISCUSSION AND SIMULATIONS}

Generally, base function specifies precision, the reference trajectory specifies the closed loop dynamic response and the coincidence point specifies robustness and a certain smoothness of the controller output (Ernst et al., 1996). For the control law (16) and (22), the coincidence point $H_{i} T_{s}$ should be at least larger than the time delay of the system or the period during which the output exhibits an inverse response (non-minimum phase). Here, the relation between the coincidence point $H_{i} T_{s}$ and system performance is discussed through simulations. Consider the integrating process (Majhi and Atherton, 1999) 


$$
G(s)=\frac{K}{s(T s+1)} e^{-\theta s}
$$

where the process parameters are $K=1, T=4, \theta=5$. The process model is built with $N=30$, the desired closed loop time is chosen as $T_{r e f}=1$, and the sampling time is set as constant $T_{s}=1$. A unit step input and a step disturbance with magnitude 0.2 at the process output are added at $t=0$ and $t=45$, respectively. Consider the case that one base function is adopted. The different $H=H_{1}$ is considered in the simulation. Responses of nominal systems are shown in Fig. 2, which indicates that faster responses of the setpoint tracking and disturbance rejection are obtained with decreasing $H$, while the manipulated variables become worse.

Generally, the coincidence point $H T_{s}$ should be chosen reasonably so that an optimal compromise between the robustness and performance is reached. The larger the coincidence point $H T_{s}$, the worse the nominal performance and the better the robustness. Assume that there exist uncertainties in model parameters with $T=3$ and $\theta=7$. Step responses of the system are shown in Fig. 3, which indicates that the proposed algorithm can provide better robustness while the parameter perturbations are taken.

\section{CONCLUSIONS}

A predictive functional control algorithm of integrating process based on impulse response model is given in this paper. By introducing the novel error compensation method, the proposed algorithm can achieve the zero steady-state error for the control system, which can also improve the disturbance rejection. Furthermore, the structure and the tuning of the given controller are very simple. Simulations show that the presented algorithm can achieve better closed loop performance and provide better robustness.
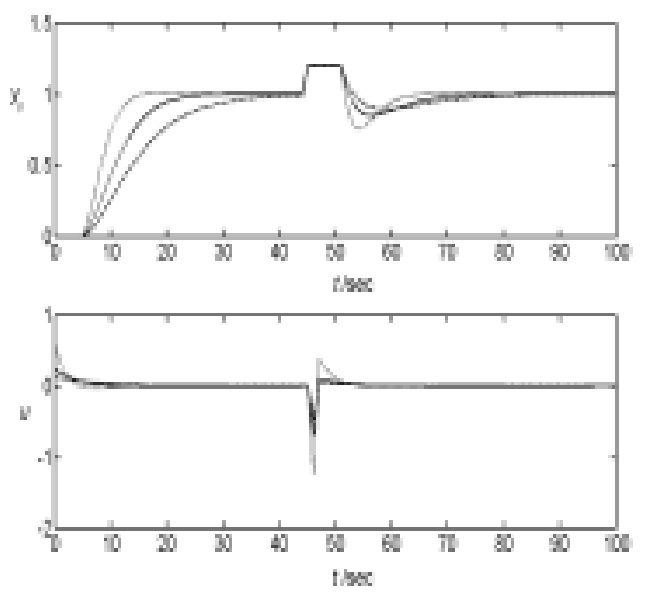

Fig. 2. Output (top) and input (bottom) of the closedloop step responses $(H=9$, dotted line; $H=12$, solid line; $H=16$, dashed line).
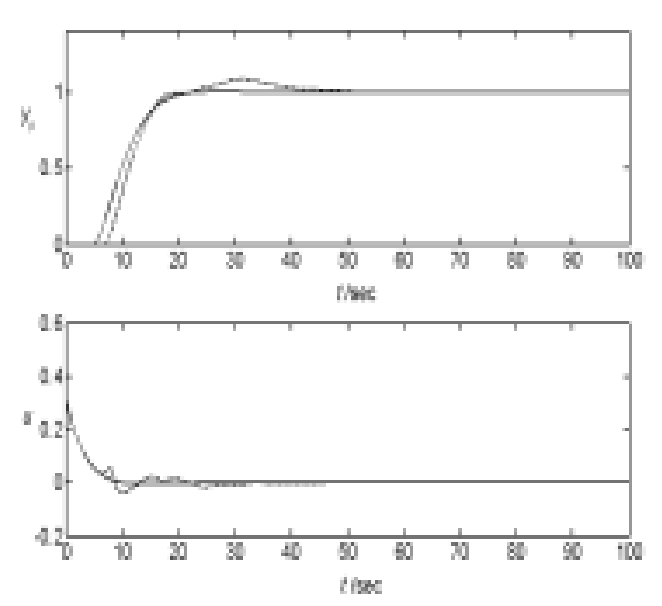

Fig. 3. Step responses of the system with $H=11$ (nominal system, solid line; uncertain system dashed line) .

\section{REFERENCES}

Abu el Ata-Doss S., Finai P. and J. Richalet (1991). Handing input and state constraints in predictive functional control. Proceeding of the $30^{\text {th }}$ conference on decision and control, Brighton, England, 985-990.

Cutler C.R. and B.1.Ramaker (1980). Dynamic matrix control-A computer control algorithm, Proc Joint Automatic Control Conference, San Francisco, Wp5-B.

Ernst T.E.F.H.C., I.H.B. Verbruggen, I.A. J. J. Boom and J. Richalet (1996). First principle modeling and predictive functional control of enthalptic processes. Delft University of Technology (France),

Majhi S. and D.P. Atherton (1999). Modified smith predictor and controller for processes with time delay. IEE Proc.-Control Theory Appl., 140(5): 359-366.

Richalet J. (1993). Industrial application of model based predictive control. Automatica, 29(5): 1259-1274.

Rossiter J.A, and J. Richalet (2002). Handing constraints with predictive functional control of unstable processes Proc of American Control Conference, Anchorage, AK, 4746-4751.

Rouhani R. and R.K. Mehra (1982). Model algorithm control (MAC): basic theoretical properties. Automatica, 18(4): 401-414.

Xi Y.G. (1991). Predictive Control. National Defence Industry Press, Beijing.

Zhang W.D., X.M. Xu and Y.X. Sun (1999). Quantitative performance design for integrating processes with time delay. Automatica, 35: 719-723.

Zhang Z.J. and Y.X. Sun (2001). Predictive control algorithm of integrating plant based on stepresponse. Control and Decision (In Chinese), 16(3): 378-379. 